\title{
HEART DISEASE IN THE ZULU: ESPECIALLY CARDIOMYOPATHY AND CARDIAC INFARCTION
}

\author{
BY \\ J. E. COSNETT \\ From the Edendale Hospital, Pietermaritzburg, Natal, South Africa
}

Received June 19, 1961

Interest in African cardiology has been focused on its two main peculiarities. One is the welldocumented rarity of coronary thrombosis. The other is the prevalence of cardiomyopathies of unknown origin among indigenous Africans. While both are receiving much publicity their extent has been difficult to assess. In the relatively undeveloped areas, where their incidence might be of greatest interest, no accurate sources of information regarding morbidity and mortality exist. In the urban areas, where such statistics are more accurate, the African population at risk is to a certain extent selected since many of its members are part of a young, migrant labour force whose homes are in native reserves away from the cities. When these people are overtaken by old age or chronic illness they return to these homes. Their fates often escape recording.

The fallacies of hospital morbidity statistics are obvious. Nevertheless, a hospital whose patients are broadly representative of the population at risk can probably contribute to the knowledge of disease incidence, especially where the difference in incidence of certain diseases is very large. Edendale Hospital enjoys advantages in this respect. Its scope and situation allow it to admit African and Indian patients of all classes. A random survey has revealed that about 5 per cent of its patients are resident in the city of Pietermaritzburg and larger towns. Approximately 37 per cent come from periurban townships and locations, and 58 per cent are truly rural in that they live in native reserves or on farms. Because the few surveys that have been made have been concentrated on the larger cities, and because these have shown certain discrepancies, we have made a detailed analysis of patients admitted to this hospital with heart disease.

\section{MATERIAL AND MethodS}

This series consists of 1,000 consecutive African patients, over 8 years old, with heart disease. They were admitted to the medical unit of this hospital over a period of 22 months from June, 1959, to March, 1961. During this time approximately 28,300 patients of all types (excluding maternity) were admitted to hospital. Of these, 25,200 (89\%) were African, principally Zulu. The remaining 3,100 $(11 \%)$ were Indian.

Diagnosis of heart disease was based on history, clinical examination, radiography, and electrocardiography. With the facilities and experience available every effort was made to arrive at precise diagnoses. On discharge from hospital each patient was classified according to age, sex, and diagnosis. In the latter classification strict criteria were observed and any patient who did not conform to one of the commonly accepted categories of heart disease was relegated to a group named "heart disease of uncertain origin." This group was subsequently analysed further. Patients who died were subjected to post mortem examination whenever this was permitted.

The following criteria were used in allocating patients to diagnostic groups.

(1) Rheumatic heart disease: includes active rheumatic fever with carditis, mitral and tricuspid 
stenosis, organic mitral and tricuspid regurgitation, aortic stenosis and aortic regurgitation in which the clinical features suggested that the cause was rheumatic heart disease.

(2) Hypertensive heart disease: a group including patients with a sustained blood pressure of over $180 / 100 \mathrm{~mm}$. $\mathrm{Hg}$ who had left ventricular or congestive cardiac failure and/or clinical and electrocardiographic evidence of left ventricular hypertrophy. A few patients with lower blood pressures were admitted to this group on account of retinal changes or post mortem findings indicating longstanding, but possibly "failed," hypertension. This group includes hypertension from all causes.

(3) Syphilitic heart disease: was diagnosed when a patient had lone aortic regurgitation and a positive Wassermann reaction or in whom the clinical bias strongly favoured this diagnosis.

(4) Tuberculous pericarditis: patients allocated to this category had constrictive pericarditis, or a serous or sero-sanguinous pericardial effusion which either responded to specific therapy or was proved to be tuberculous at necropsy.

(5) Cor pulmonale: signified patients in congestive cardiac failure without cause other than definite clinical, radiological, or pathological evidence of chronic advanced lung disease, usually chronic bronchitis and emphysema, bronchiectasis or pulmonary fibrosis.

(6) Myocardial infarction: patients in this group either had unequivocal electrocardiographic evidence of infarction or proof of diagnosis was obtained at necropsy.

(7) In other specific conditions (non-tuberculous pericarditis, congenital heart disease, anæmias, kyphoscoliotic heart disease, and primary arrhythmias) it was usually not difficult to classify the patients on prima facie evidence.

(8) Cardiac failure of uncertain origin: a substantial number of patients who could not be relegated to any of the above groups were admitted to this category.

\section{Diagnostic Difficulties}

It is characteristic of medical practice among Africans that there is a large number of cardiac patients who do not show the kinds of disease encountered commonly among Europeans. The newcomer to Africa is often bewildered by the extent of this problem. Gradually, as experience advances, a common pattern becomes apparent among most of the patients with idiopathic heart failure. Similarly, in the present series, over one-half of the cases which were not initially classifiable into the commonly accepted cardiological categories showed distinct features which enabled one to recognize them as a separate group. These features are identical with those which have been described in several centres in South Africa by various authors and by diverse names. Gillanders (1951) called the condition "nutritional heart disease." Becker et al. (1953) are of the opinion that it is a cardiovascular collagenosis. Others have described it as cardiomyopathy of unknown origin, South African cardiomyopathy, or cryptogenic heart disease. The name is of little importance for the cause remains obscure. What is significant is that there is a form of heart disease common among South African natives that is distinct from the usual European cardiac disorders, and which is clinically and pathologically recognizable. My experience of these common South African cardiomyopathies leads me to believe that they are fundamentally one disease, but that their clinical presentation and course varies considerably. This variation led to some initial confusion when the conditions were first described. At a symposium held in Johannesburg the chairman (McGregor, 1957) reached a similar conclusion. The contributors to this symposium agreed that the South African cardiomyopathy differs from the endomyocardial fibrosis which is prevalent in Central and East Africa.

In this analysis, having separated the typical cardiomyopathies, there remains a group of patients who are still unclassified. It is this group which has provided the greatest diagnostic uncertainty. Most cases are probably also examples of cardiomyopathy, since it is inherent in the nature of the disease that it may mimic several of the other forms of heart disease encountered in this and in European communities. Discussion of examples of these will not only clarify these difficulties but will also illustrate some clinical features of the cardiomyopathy and its differential diagnosis. 
Hypertensive Heart Disease. It is a common observation that many patients with heart failure due to cardiomyopathy have high blood pressures on admission to hospital. This leads to some being erroneously diagnosed as hypertensive heart disease, especially when there is some evidence of left ventricular hypertrophy. This mistake usually becomes obvious when the blood pressure falls within a few days and when the patient is found to have no retinal signs of hypertension. More difficult is the case in which the blood pressure remains moderately elevated and which has mild retinal changes. Such patients, in whom it was found impossible to decide whether heart failure was caused by hypertension or cardiomyopathy, or a combination of both, are included in the group of doubtful ætiology.

Mitral Regurgitation. The majority of cases of cardiomyopathy in congestive cardiac failure have pansystolic apical murmurs of varying intensity. That these are due to functional mitral incompetence consequent on cardiac dilatation has been repeatedly shown at necropsy. In some instances the signs of mitral incompetence diminish or disappear during treatment. It is in the case in which the signs are persistent that one is tempted to diagnose organic mitral disease, frequently to be corrected at necropsy when no organic valve lesion is found. The difficulty in deciding clinically whether mitral regurgitation is organic or functional has contributed more cases to the doubtful group than any other cause. Yet, if we have erred in classifying them thus, the bias has been in the direction of reducing the number of cases classified definitely as cardiomyopathy.

Pericardial Effusion. Some of the most severe cases of congestive heart failure due to cardiomyopathy are admitted to hospital with grossly dilated, inactive hearts which produce low voltage cardiograms with $\mathrm{T}$ wave flattening or inversion. X-ray appearances are often equivocal. The absence of a pericardial friction rub does not necessarily exclude a diagnosis of pericarditis with effusion and the likelihood of erroneously making this diagnosis is great. The only certain method of eliminating the possibility of a pericardial effusion is by paracentesis, a procedure which we have performed on several patients without finding fluid.

Constrictive Pericarditis. Mindful of the fact that 38 per cent of patients with constrictive pericarditis have heart shadows which are moderately or greatly enlarged on fluoroscopy (Wood, 1956), we have occasionally experienced difficulty in deciding whether a patient with a large inactive heart has constrictive pericarditis or cardiomyopathy. A negative paracentesis might only mean that the large heart shadow is caused by a grossly thickened pericardium such as has been found at necropsy in some cases. The only way of showing the correct diagnosis is by right atrial catheterization or angiocardiography.

Cor Pulmonale. Chronic lung diseases are common in this community. The coexistence of emphysema or pulmonary fibrosis with heart failure leads one to suspect that the latter is the result of the former. This is often the case. Sometimes, however, one cannot be sure to what extent this relationship exists. One feels in some instances that a heart which is predisposed to cardiomyopathy may fail at an earlier stage because of the added burden of chronic lung disease. Such cases of "cardiomyopathy with an element of cor pulmonale" are included in the doubtful group.

Beri-beri. From Cape Town Schrire $(1959,1960)$ has described a number of cases of beri-beri heart disease. The possibility exists that certain of our cases of unexplained heart failure are caused by beri-beri. These must, however, be rare since a hyperkinetic circulation is not a feature of our cases of idiopathic heart failure. Moreover, they do not respond to treatment with aneurine in large doses by injection, and the natural history of the condition is unlike that of beri-beri. The difference in incidence between Natal and the Western Cape may be due to the rarity of chronic alcoholism among the Zulu. Though no typical examples have been seen there are a few who might have had beri-beri. These have been included in the doubtful group.

Heart Failure in the Aged. Many patients in whom heart failure was never adequately diagnosed were over 60 years old. Some of these had intercurrent infections of the respiratory, urinary or gastrointestinal systems. They had in addition signs of congestive cardiac failure for which no cause was ever apparent. It is possible that the term "degenerative heart disease" or "toxic myocarditis" might have been applied to some of them but we have no alternative but to include 
these cases in the doubtful group. It seems likely that these were examples of hearts prone to cardiomyopathy in which failure occurred under the load of age or intercurrent illness. At necropsy endocardial thrombosis has been found in some of them. This endocardial thrombosis, usually at the apex of one or both ventricles, has come to be regarded as one of the specific pathological features of cardiomyopathy.

Thus, in this large group of patients, it was found impossible to ascribe the heart disease to any one cause. It appears likely that in some cases more than one cause was operating and it may have been the combined effect of hypertension, chronic lung disease, infection, or old age on a myocardium which was the seat of some intrinsic defect that ultimately produced heart failure.

\section{RESULTS AND DisCUSSION}

The results of the analysis of 1,000 consecutive cases are shown in Table I. The incidence and age and sex distribution of hypertensive and rheumatic heart disease are not remarkable and resemble those described in other series of African and European patients (Mackay, 1951; Schwartz

TABLE I

Analysis of Aetiology among 1,000 Consecutive Cases of Heart Diseases in Africans

\begin{tabular}{|c|c|c|c|c|c|c|c|c|c|c|c|c|c|c|c|c|c|c|c|}
\hline \multirow{2}{*}{ Disease } & \multirow{2}{*}{$\begin{array}{l}\text { Age } \\
\text { Sex } \\
\end{array}$} & \multirow{2}{*}{$\cdots$} & \multicolumn{2}{|c|}{$8-19$} & \multicolumn{2}{|c|}{$20-29$} & \multicolumn{2}{|c|}{ 30-39 } & \multicolumn{2}{|c|}{$40-49$} & \multicolumn{2}{|c|}{$50-59$} & \multicolumn{2}{|c|}{$60-69$} & Over 70 & \multirow{2}{*}{$\begin{array}{l}\text { Total } \\
\text { male }\end{array}$} & \multirow{2}{*}{$\begin{array}{c}\text { Total } \\
\text { female }\end{array}$} & \multirow{2}{*}{ Total } & \multirow{2}{*}{$\%$} \\
\hline & & & & & $\mathbf{M}$ & $\mathbf{F}$ & & & $\mathbf{M}$ & & & & $\mathbf{M}$ & & $\mathbf{M} \mathbf{F}$ & & & & \\
\hline Hypertensive heart disease & $\cdots$ & $\cdots$ & 1 & 1 & 5 & 8 & 16 & 26 & 17 & 32 & 18 & 15 & 16 & 21 & $11 \quad 15$ & 84 & 118 & 202 & $20 \cdot 2$ \\
\hline Rheumatic heart disease & $\cdots$ & $\ldots$ & 20 & 39 & 15 & 18 & 15 & 25 & 21 & 18 & 8 & 9 & 2 & 8 & 3 & 84 & 121 & 205 & $20 \cdot 5$ \\
\hline Syphilitic heart disease & $\cdots$ & $\cdots$ & 0 & 0 & 2 & 0 & 6 & 4 & 18 & & 9 & 3 & 13 & 6 & 1210 & 60 & 28 & 88 & $8 \cdot 8$ \\
\hline Tuberculous pericarditis & $\cdots$ & $\ldots$ & 1 & 1 & 4 & 1 & 2 & 2 & 3 & 3 & 2 & 0 & 1 & 0 & 0 & 14 & 7 & 21 & $2 \cdot 1$ \\
\hline Cor pulmonale .. & . & $\cdots$ & 0 & 1 & 0 & 0 & 13 & 2 & 26 & 5 & 34 & 9 & 30 & 12 & $20 \quad 8$ & 123 & 37 & 160 & $16 \cdot 0$ \\
\hline Bacterial endocarditis .. & $\cdots$ & $\cdots$ & 2 & 5 & 0 & 3 & 3 & 2 & 7 & 2 & 1 & 1 & 4 & 0 & 0 & 17 & 13 & 30 & $3 \cdot 0$ \\
\hline Cardiomyopathy & $\cdots$ & $\cdots$ & 2 & 3 & 6 & 19 & 16 & 21 & 23 & 10 & 14 & 11 & 5 & 8 & 00 & 66 & 72 & 138 & $13 \cdot 8$ \\
\hline Other heart disease of uncerta & ain ori & $\operatorname{gin}$ & 0 & 0 & 2 & 0 & 8 & 6 & 4 & 7 & 16 & 16 & 17 & 15 & $26 \quad 16$ & 73 & 60 & 133 & $13 \cdot 3$ \\
\hline Myocardial infarction .. & $\cdots$ & $\cdots$ & 0 & 0 & 1 & 0 & 1 & 0 & 1 & 0 & 2 & 0 & 1 & 0 & 0 & 6 & 0 & 6 & 0.6 \\
\hline Congenital heart disease & $\cdots$ & $\cdots$ & 3 & 2 & 0 & 0 & $\mathbf{0}$ & 0 & 1 & 0 & 0 & 0 & 0 & 0 & 0 & 4 & 2 & 6 & 0.6 \\
\hline Non-tuberculous pericarditis & $\cdots$ & $\cdots$ & $\mathbf{0}$ & 0 & 1 & 0 & $\mathbf{0}$ & 0 & 2 & $\mathbf{0}$ & $\mathbf{0}$ & 0 & 1 & 0 & 00 & 4 & 0 & 4 & 0.4 \\
\hline Kyphoscoliotic heart disease & $\cdots$ & $\cdots$ & 1 & 1 & 0 & 0 & 0 & 0 & 0 & 0 & 0 & 0 & 0 & 0 & 0 & 1 & 1 & 2 & 0.2 \\
\hline Anæmic heart disease .. & $\cdots$ & $\cdots$ & 0 & 0 & 0 & 2 & 0 & 1 & 0 & 0 & 0 & 0 & 0 & 0 & 0 & 0 & 3 & 3 & $\mathbf{0 . 3}$ \\
\hline \multirow[t]{2}{*}{ Primary arrhythmias } & $\cdots$ & . & 0 & 0 & 0 & 1 & 0 & 0 & 1 & 0 & 0 & 0 & 0 & 0 & $0 \quad 0$ & 1 & 1 & 2 & 0.2 \\
\hline & & & & & & & & & & & \multicolumn{2}{|c|}{ Total } & . & & . & 537 & 463 & 1000 & 100 \\
\hline
\end{tabular}

et al., 1958; Schrire, 1960). The high incidence of syphilitic heart disease is paralleled by the frequency of meningovascular syphilis in this community. In comparison with other series the prevalence of cor pulmonale is surprising. It is probably explained by the frequency of chronic respiratory disease in which treatment is often delayed. Furthermore, patients with chronic heart disease of this type tend to gravitate to the rural areas where they need increasingly frequent readmission to hospital. Among the less common varieties of heart disease both tuberculous pericarditis and bacterial endocarditis carry a high mortality.

Notable rarities in this series are cases of myocardial infarction, ischæmic heart disease, thyrotoxicosis and congenital heart disease. Previous authors have varied in their impressions of the frequency of congenital heart disease in the Bantu. Its rarity in this series is partly explained by the omission of patients under 8 years of age. Local factors such as the inadequacy of obstetric and neonatal care in the rural districts, and the fact that survival depends largely on innate fitness, might account for our observation that fewer of these patients reach hospital than would be expected. 
Nevertheless one is left with the distinct impression that congenital heart disease is rarer in the Zulu than in other comparable races in this region (European and Indian). It is universally agreed that thyrotoxicosis is very rare in the South African Bantu races. During the period occupied by this survey we have seen only one doubtful case of thyrotoxicosis and this patient had no cardiac involvement.

Cardiomyopathy and Cardiac Failure of Uncertain Origin. Though these conditions have received wide attention in Africa and most of their clinical features are well documented, this analysis of our experience reveals facets that we feel have not been sufficiently emphasized. Table I shows that definite cardiomyopathies and cardiac failure of uncertain origin account for 27 per cent of cases of heart disease admitted to hospital, a larger proportion than is accounted for by any other cause. In about one-half of these it was possible to diagnose cardiomyopathy with a degree of certainty which avoided incriminating any other cause. A large proportion of the remainder were probably suffering from a similar disease in varying degrees of severity but were classified separately for the sake of accuracy because it was felt that the diagnosis was in doubt. In some instances another cause may have contributed to cardiac failure in a patient whose heart was already weakened by what we call cardiomyopathy. This interpretation is especially applicable to older patients in whom coincident causes of heart disease are more likely to be present.

A study of the age incidence of cardiomyopathy shows that in the younger age groups the disease predominates among women. This preponderance coincides with the child-bearing age. Whether or not child-bearing has a direct effect in causing cardiomyopathy is open to argument. It is particularly noteworthy that many of these young women who develop cardiomyopathy do so within six months of childbirth though the condition has occasionally been seen in young nulliparæ. Clinically and pathologically this postpartal cardiomyopathy appears indistinguishable from that which occurs among older women and among men. Other authors in South Africa have commented similarly on its occurrence and clinical features (Seftel and Susser, 1961; Reid, 1961), but our experience makes us consider the prognosis of postpartal cardiomyopathy to be as grave as in the disease which occurs in males and postmenopausal women. Several deaths and numerous cases of intractable cardiac failure have occurred among our postpartal patients. A peculiar feature is that the cardiomyopathy of the postpartum period confines its appearance to this time. One would expect that, in common with other heart diseases, the heart would be most likely to fail when it is carrying its greatest load in the last three months of pregnancy. Yet this disease usually follows an entirely normal pregnancy and confinement and we have never yet had occasion to diagnose heart disease of this type during pregnancy. It appears that, whatever its cause, the myocardial weakness does not develop until after delivery, a fact which is both strange and tantalizing. The prevalence of this type of heart disease among young mothers weighs against the theory, held by some, that the cardiomyopathy is a manifestation of ischæmic heart disease. For this is precisely the age group and sex in whom ischæmic heart disease is least expected.

Endocardial thrombosis has been described by most observers of South African cardiomyopathy as well as in other cardiomyopathies. Our experience leads us to regard this endocardial thrombosis in one or both ventricles, usually at the apex, as one of the most consistent pathological features of cardiomyopathy. It does not occur only as a sequel to prolonged cardiac failure and its development seems to be one of the earliest macroscopic events. It has sometimes been observed in patients in whom there was no evidence of heart disease before death from a noncardiac cause. It is not associated with underlying myocardial infarction. A further feature which deserves mention because it has been insufficiently emphasized is the occurrence of visceral infarction by emboli which presumably arise from the endocardial thrombosis. Pulmonary embolism occurs more commonly in cardiomyopathy than it does as a result of femoral vein thrombosis which is a rare condition in the Bantu. At post mortem examination infarcts of the spleen and kidneys are often found in cases which have adherent endocardial thrombosis. Clinically we have observed that the progress of patients with cardiomyopathy, especially the most severely affected, is sometimes marred by episodes of unexplained shock and peripheral vascular failure. A few of these cases 
have signs of pulmonary embolism. In others we have suspected that the explanation of such episodes is recurrent embolization of the abdominal organs, evidence of which may be seen at necropsy. Two of our patients suddenly developed hemiplegia while under treatment for cardiomyopathy. In a further case a woman with severe intractable cardiac failure suddenly developed signs of femoral artery occlusion and subsequently gangrene of the leg. These instances of flagrant or suspected embolism have usually occurred independently of any cardiac arrhythmia. Arrhythmias of all types are uncommon in association with cardiomyopathy.

Myocardial Infarction. During the period occupied by this survey myocardial infarction has been diagnosed six times. All these patients were men. Rigid standards of diagnosis were applied, though the problem, common in European practice, of deciding whether or not a patient has suffered a myocardial infarction does not often arise. With the single exception of cases of syphilitic aortitis anginal pain is very uncommon. One rarely sees the cardiographic changes which in the European are indicative of myocardial ischæmia.

Three patients were shown to have myocardial infarction at necropsy. The remaining three had cardiographic changes which were considered unequivocal: namely, deep $\mathrm{Q}$ waves, elevated $\mathrm{S}-\mathrm{T}$ segments, and typical $\mathrm{T}$ wave inversion in anterior chest leads (1 case); or in leads reflecting the posterior surface of the heart ( 2 cases). Serum cholesterol estimations were done in four cases. None was elevated. One patient was a diabetic. Of those who were examined at necropsy only one showed a moderate degree of atherosclerosis. One other interesting patient was a man of 26 years who had been admitted to hospital repeatedly in congestive cardiac failure with a grossly dilated heart. The cardiogram showed left bundle-branch block. The clinical diagnosis was cardiomyopathy. At necropsy he had a dilated heart, functional mitral incompetence and apical endocardial thrombosis; in addition, there was a septal infarct, proved microscopically, without coronary atherosclerosis. We presumed that the infarct had originated from a small embolus which had occluded a coronary vessel. Myocardial infarction has not been diagnosed in a Zulu woman during this series.

Comparison with Indians. Edendale Hospital is fortunate in being able to admit, and compare the incidence of disease in, two races (Bantu and Indian) among whom there is no racial admixture. During the period occupied by this survey approximately 3,100 Indian patients were admitted to the general wards. Of these at least eleven suffered from definite myocardial infarction, while only one case has been seen in which the cause of heart failure in an Indian was thought to be cardiomyopathy. The Indians resemble the European population in that heart disease among them follows the same pattern and is rarely "idiopathic." From our hospital experience therefore it appears that myocardial infarction is at least 15 times more common in the Indian than in the Zulu. Cardiomyopathy on the other hand is from 16 to 32 times more common in the Zulu than in the Indian.

Comparison with Surveys from Other Areas. Clinical studies of the incidence of heart disease in the South African Bantu have been made in Cape Town, Johannesburg and Durban. All these are predominantly urban areas and none of the previous studies has included as many Bantu cardiac patients as have been analysed in the present study. The results of our survey resemble those published from Johannesburg by Schwartz et al. (1958) and from Durban by Adams and Wainwright (1958). The discrepancies that have occurred, notably the greater incidence of cor pulmonale and syphilitic aortitis at Edendale, probably reflect the age structure of the populations at risk, and the tendency for older patients with chronic illnesses to return to the rural areas. Schrire (1960) in Cape Town found a greater incidence of pericarditis and beri-beri than we have diagnosed, and it appears that cardiomyopathy is much less prevalent in the Western Cape than it is in Natal or in the Transvaal. Bantu heart disease in the latter two provinces seems similar, whereas heart disease in the Cape has a slightly different complexion.

Myocardial infarction and ischæmia are universally rare among the South African Bantu. The age and sex incidence of cardiomyopathy make it unlikely that this disease is directly related to coronary vascular disease as we see it among the European and Indian populations of South 
Africa. Indeed, the incidence of cardiomyopathy is so different from that of coronary disease that one might postulate that the two conditions are "complementary" in that the former is due to a deficiency of factors which, in excess, predispose to the latter. We have not found any association between cardiomyopathy and any known nutritional deficiency, though the possibility of deficiency of some unknown factor (such as a trace metal, amino-acid, or fatty acid) has not yet been excluded. A toxic dietary factor might also be incriminated.

\section{SUMMARY AND CONCLUSIONS}

A survey of 1,000 consecutive patients with heart disease admitted to hospital has revealed the rarity of myocardial infarction and the high incidence of heart disease of unknown origin among the Zulu population of inland Natal. The broad pattern of heart disease in this area resembles that in Johannesburg and Durban but differs somewhat from that in the Western Cape Province. The cardiomyopathy that is common among the native races in most parts of South Africa is probably a single disease entity in which the clinical picture and course is, nevertheless, extremely variable. It differs in many respects from the endomyocardial fibrosis of Central and East Africa.

Attention is drawn to the frequency of cardiomyopathy in the post-partum period, and to the common association of endocardial thrombosis and visceral infarction in cases of cardiomyopathy.

I thank the Medical Superintendent, Dr. T. M. Adnams, for permission to publish, and Miss C. Hinde for help with the case records.

\section{REFERENCES}

Adams, E. B., and Wainwright, J. (1958). Brit. med. J., 2, 914.

Becker, B. J. P., Chatgidakis, C. B., and van Lingen, B. (1953). Circulation, 7, 345.

Gillanders, A. D. (1951). Brit. Heart J., 13, 177.

McGregor, M. (1957). "Report of a Joint Seminar of the Departments of Pathology and Medicine of the University of the Witwatersrand." S. Afr. med. J., 31, 854.

Mackay, D. (1951). Hospital Morbidity Statistics: A preliminary study of inpatient discharges. H.M. Stationery Office, London.

Reid, J. V. O. (1961). S. Afr. med. J., 35, 165.

Schwartz, M. B., Schamroth, L., and Seftel, H. C. (1958). Med. Proc., 4, 275.

Schrire, V. (1959). C. Afr. J. med., 5, 349. (1960). Amer. Heart J., 59, 835.

Seftel, H., and Susser, M. (1961). Brit. Heart J., 23, 43.

Wood, P. (1956). Diseases of the Heart and Circulation. Eyre and Spottiswoode, London. 